\title{
Bayesian modelling of a thermal helium beam for measure- ment of electron density and temperature in the W7-X diver- tor plasma
}

Maciej Krychowiak ${ }^{1}$, Mathias Brix ${ }^{2}$, Dirk Dodt ${ }^{1}$, Yühe Feng ${ }^{1}$, Ralf König ${ }^{1}$, Oliver Schmitz , Jakob Svensson $^{1}$ and Robert Wolf ${ }^{1}$

${ }^{1}$ Max-Planck-Institut für Plasmaphysik, EURATOM Association, D-17491 Greifswald, Germany

${ }^{2}$ Culham Centre for Fusion Energy, EURATOM/CCFE Association, Culham Science Centre, Abingdon, Oxfordshire, OX14 3DB, UK

${ }^{3}$ Institut für Energieforschung (Plasmaphysik), FZ Jülich, EURATOM Association, TEC, D52428 Jülich, Germany

\section{Abstract}

In Greifswald/Germany W7-X, a new stellarator-type fusion plasma experiment is currently being built. For the investigation of the divertor plasma two thermal helium beams are foreseen. This diagnostic is routinely used on several fusion plasma experiments and is capable of measuring radial profiles of electron density and temperature with good spatial and temporal resolution in the range of typical edge plasma parameters $n_{e}=10^{18}-10^{19} \mathrm{~m}^{-3}$ and $T_{e}=20-200$ $\mathrm{eV}$. The penetration depth of the beam is limited by electron collisional ionisation of the helium atoms and amounts to $3-8 \mathrm{~cm}$ in this parameter range. In this paper we investigate the beam propagation for detached plasma conditions in the $\mathrm{W} 7-\mathrm{X}$ divertor region (based on a background plasma simulated with the 3D plasma and neutral transport code EMC3/EIRENE), in which the electron density in the divertor may well exceed $10^{20} \mathrm{~m}^{-3}$, as observed in the predecessor experiment W7-AS. In this regime the beam penetration drops to $1-2 \mathrm{~cm}$. Through 
a Bayesian approach, we include uncertainties of all rate coefficients for electronic excitation and ionisation used in the collisional-radiative model of atomic helium based on a steady-state approximation valid for a relaxed thermal or supersonic beam. Bayesian inversion of simulated signals for $\mathrm{W} 7-\mathrm{X}$ conditions provides a reliable quantitative estimation of the propagation of uncertainties of the atomic data to the $\mathrm{n}_{\mathrm{e}}$ and $\mathrm{T}_{\mathrm{e}}$ errors as well as input for potential improvements of the diagnostic setup. For example, the temperature error at $\mathrm{T}_{\mathrm{e}}=5 \mathrm{eV}$ and $\mathrm{n}_{\mathrm{e}}=10^{20} \mathrm{~m}^{-3}$ can be reduced from app. $50 \%$ to $9 \%$ by absolute calibration of the observation system and fitting of three absolute line intensities instead of two line intensity ratios to the model.

\section{Introduction}

Thermal and supersonic helium beams are widely used for local profile measurements of the electron density $n_{e}$ and electron temperature $T_{e}$ in the edge region of magnetically confined fusion plasmas ([1], [2], [3] and references therein). Typically, an accuracy of around $10 \%\left(\mathrm{n}_{\mathrm{e}}\right)$ and $30 \%\left(\mathrm{~T}_{\mathrm{e}}\right)$ can be achieved [1], [3]. The plasma parameters are derived by comparing measured intensity ratios of three spectral lines of atomic helium with model calculations. The applied collisional-radiative (CR) model contains more than one hundred rate coefficients for electron collisional excitation and ionisation of atomic helium in a plasma. Such comprehensive sets of rate coefficients are only available from numerical calculations. As experimental validations are available for a small fraction of the transitions (e.g. [4], [5], [6], [7]), the atomic data is subject to relatively large uncertainties. Due to the complexity of the CR model it is very difficult to quantify the impact of uncertain rate coefficients on the errors of $\mathrm{n}_{\mathrm{e}}$ and $\mathrm{T}_{\mathrm{e}}$ [3], [8]. Hence, we developed a probabilistic approach based on Bayes' theorem which allows quantitative investigation of the propagation of uncertainties of atomic data to the errors of derived plasma parameters.

In Greifswald (Germany), the optimized stellarator W7-X is currently under construction. In its divertor region very high electron densities are expected in the partially detached plasma regime, based on the observations at the stellarator experiment W7-AS [9]. The main aim of this work is to study the suitability of the diagnostic helium beam - i.e. the expected errors of derived $n_{e}$ and $T_{e}$ values - at the 
high density and low temperature of the W7-X divertor plasmas. For this we have implemented a synthetic diagnostic based on the Bayesian inversion of simulated signals for the conditions expected in the W7-X divertor. In the next step it is planned to apply this analysis model to the supersonic helium beam diagnostic newly installed at the stellarator WEGA in Greifswald [10]. Furthermore, the probabilistic approach allows incorporating information about model parameters from additional independent sources. We give an example of how a comparison of the helium beam with other diagnostics can be used to find a set of effective rate coefficients allowing improved $n_{e}$ and $T_{e}$ reconstruction.

\section{Helium beam excitation model}

The results presented in this paper have been obtained by use of two different versions of the CR model for the helium beam emission: based on the time-dependent and the steady-state solution. In both of them we consider the ground and 28 excited states (up to the $n=5$ shell) of atomic helium [11]. The simulation of the beam penetration through the plasma and its attenuation due to ionisation losses (Figure 2 (a)) is obtained by solving the time-dependent system of balance equations for all 29 levels. The population of the level $i$ depends on the electron collisional excitation and deexcitation, spontaneous emission and electron collisional ionisation:

$$
\frac{d n_{i}}{d t}=\sum_{j \neq i}\left\langle\sigma_{j \rightarrow i} v_{e}\right\rangle n_{e} n_{j}-\sum_{j \neq i}\left\langle\sigma_{i \rightarrow j} v_{e}\right\rangle n_{e} n_{i}+\sum_{j>i} A_{j \rightarrow i} n_{j}-\sum_{j<i} A_{i \rightarrow j} n_{i}-\left\langle\sigma_{i \rightarrow i o n} v_{e}\right\rangle n_{e} n_{i} .
$$

The movement of the beam particles through the plasma is allowed for by considering the electron density and temperature as functions of (penetration) time. At each radial position we additionally compute the level populations relative to the ground level population by use of the steady-state solution of the CR model for the local electron density and temperature. This is done by setting to zero the time derivative in the balance equations. As the relative level populations calculated by the time dependent and the steady-state solution are practically the same (see Figure 2 (a) and its explanation in Section 4), we conclude that due to the high electron densities the steady-state approximation is applicable for the calculation of relative level populations. Hence, the Bayesian sensitivity analysis, show- 
ing the applicability of the diagnostic in the high density regime, is performed using the steady-state solution of the CR model which is the forward function of our Bayesian model.

\section{Probabilistic Model}

In order to study the precision of $\mathrm{n}_{\mathrm{e}}$ and $\mathrm{T}_{\mathrm{e}}$ that can be achieved for $\mathrm{W} 7-\mathrm{X}$ divertor plasmas, given uncertainties in observations and atomic cross section data, a Bayesian approach will be taken. Other examples for the application of Bayesian methods in plasma physics can be found in [12], [13], [14], [15], [16], [17], [18], [19], [20], [21], [22], [23]. The textbooks [24], [25] give comprehensive introductions of the used approach.

The investigation of the influence of uncertain atomic rate coefficients on $n_{e}$ and $T_{e}$ is done by forming the joint posterior pdf for the rate coefficients, $\theta, \mathrm{n}_{\mathrm{e}}$ and $\mathrm{T}_{\mathrm{e}}$ for a given position along the beam:

$$
p\left(n_{e}, T_{e}, \theta \mid D\right)=\frac{p\left(D \mid n_{e}, T_{e}, \theta\right) p\left(n_{e}, T_{e}\right) p(\theta)}{p(D)}
$$

where $D=\left\{r_{i}\right\}$ are the measured intensity ratios $r_{i}$. The likelihood term, $p\left(D \mid n_{e}, T_{e}, \theta\right)$, is a measure of the misfit between the intensity ratios predicted from the model parameters, and the observed ratios. It is chosen as a Gaussian distribution centred on the intensity ratios predicted by the solution to the steady-state CR model (1) with time derivatives set to zero. The priors $p\left(n_{e}, T_{e}\right)$ and $p(\theta)$ define any a priori knowledge we have about these parameters. Here $p(\theta)$ is chosen as a multivariate normal distribution with a diagonal covariance matrix, the individual variances for each rate coefficient taken from the ADAS database (see Section 5). $p\left(n_{e}, T_{e}\right)$ is chosen depending on the specific problem to be solved, see Sections 5 and 6.

The influence of the uncertainties of the rate coefficients on $n_{e}$ and $T_{e}$ can then be calculated by marginalising (2) over $\theta$ :

$$
p\left(n_{e}, T_{e} \mid D\right)=\int_{\theta} p\left(n_{e}, T_{e}, \theta \mid D\right) d \theta
$$


Since the dimensionality of $\theta$ is typically about 200 , and the posterior pdf highly nonlinear due to the solution of the rate equations (1) for each evaluation, special numerical techniques are necessary to carry out this integral. For the results presented in Section 5, we have computed the marginal posterior pdf $p\left(n_{e}, T_{e} \mid D\right)$ on a $2 \mathrm{D}$ grid in $\left(\mathrm{n}_{\mathrm{e}}, \mathrm{T}_{\mathrm{e}}\right)$ by computing the integrals

$$
p\left(n_{e}^{i}, T_{e}^{j} \mid D\right)=\int_{\theta} p\left(n_{e}^{i}, T_{e}^{j}, \theta \mid D\right) d \theta \sim p\left(n_{e}^{i}, T_{e}^{j}\right) \int_{\theta} p\left(D \mid n_{e}^{i}, T_{e}^{j}, \theta\right) p(\theta) d \theta
$$

from the expected value of the likelihood under the prior

$$
E_{\theta}\left[p\left(D \mid n_{e}^{i}, T_{e}^{j}, \theta\right)\right]=\int_{\theta} p\left(D \mid n_{e}^{i}, T_{e}^{j}, \theta\right) p(\theta) d \theta \approx \frac{1}{N_{k}} \sum_{k=1}^{N_{k}} p\left(D \mid n_{e}^{i}, T_{e}^{j}, \theta_{k}\right)
$$

where $\theta_{k}$ are samples from $p(\theta)$. This is then followed by a normalisation of the 2D marginal posterior over $n_{e}$ and $T_{e}$. A direct estimate of the error of the calculated pdf values at the grid points is also available from the spread of the samples (Figure 1 shows an example of the 2D posterior and its relative standard deviation at each grid point). The method is highly parallelisable, since each sample can be independently calculated, and easier to setup in comparison to a Markov Chain Monte Carlo run. It also has no adjustable parameters, except for the number of samples and the grid choice, which has to be assumed a priori. The chosen method is only applicable if the likelihood distribution is sufficiently broad (which it is for the current problem), and there is substantial knowledge about the prior, from which samples can be generated using standard techniques. Inferring rate coefficients as shown in Section 6 relies on the same procedure except for including $n_{e}$ and $T_{e}$ and excluding two rate coefficients of interest from the set of marginalised parameters. 

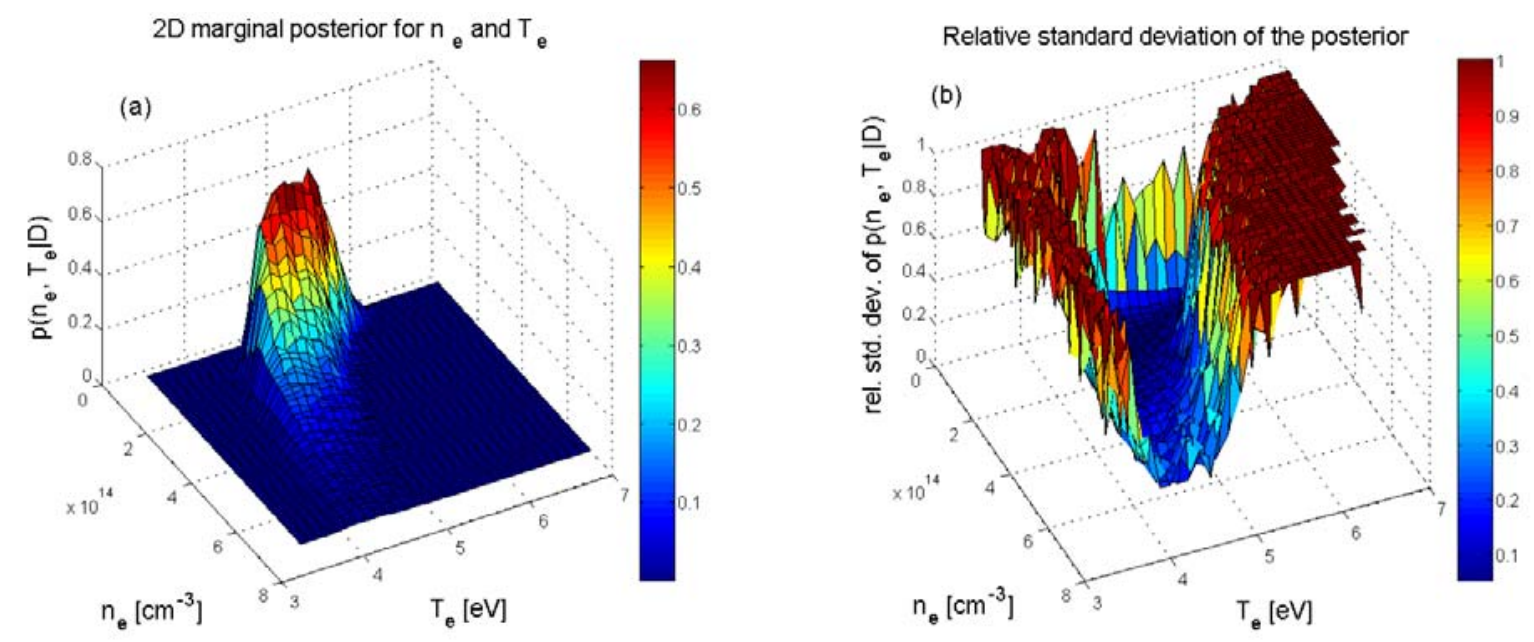

Figure 1: (a) Example of a 2D posterior distribution for $n_{e}$ and $T_{e}$. (b) Corresponding relative standard deviation of the posterior value for each grid point.

\section{Application of the helium beam at high densities in W7-X}

For W7-X the installation of two thermal helium beam diagnostics (in the lower and in the upper divertor) is envisaged [26]. Each of them consists of a nozzle box with 5 independent piezo gas valves in a poloidal arrangement in order to extend the spatial resolution to the poloidal direction. For the application of the helium beam in a divertor plasma two questions arise. The typically high electron density poses a barrier for the beam penetration since the electron collisional ionisation is the main loss process of the helium atoms in the beam. Furthermore, the high densities, typically accompanied by low temperatures, span a parameter range different from the standard helium beam application. We investigate the beam propagation in this new parameter range and assess the uncertainties of the experimental $\mathrm{n}_{\mathrm{e}}$ and $\mathrm{T}_{\mathrm{e}}$ values. 


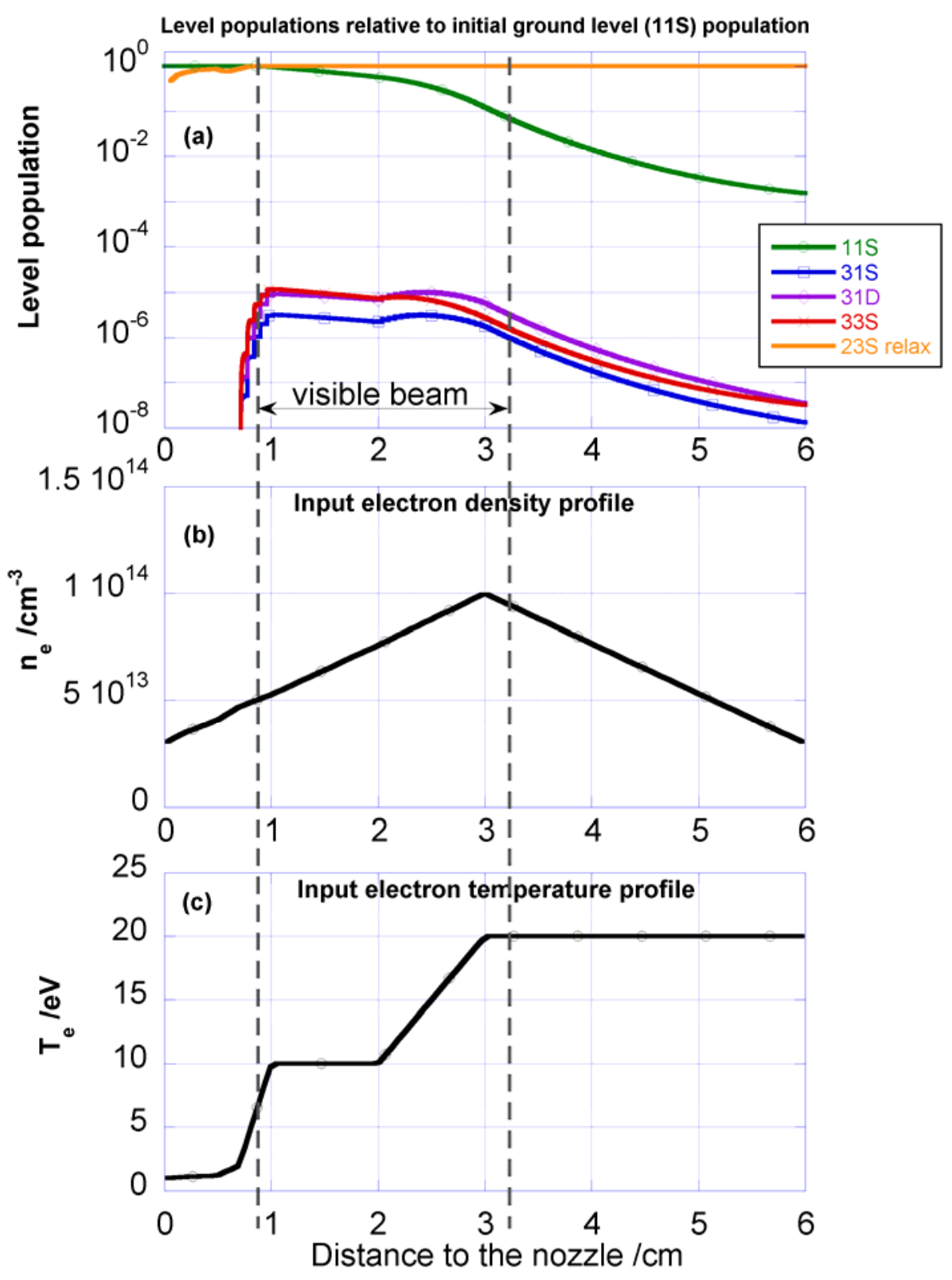

Figure 2: (a) Calculated radial profiles of level populations. "11S": ground level $\left(1^{1} \mathrm{~S}\right)$ population normalized to its initial population (at the nozzle position; the beam divergence is not accounted for). "31S", "31D" and "33S": populations (relative to the initial ground level population) of three radiating levels $3^{1} \mathrm{~S}, 3^{1} \mathrm{D}$ and $3^{3} \mathrm{~S}$, respectively. "23S relax": ratio of the relative (to the ground state) populations of the metastable level $2^{3} \mathrm{~S}$ calculated by the steady-state and the time-dependent solution. (b) and (c): profiles of electron density (b) and electron temperature (c) used for the calculation. 
The 3D plasma and neutral transport code EMC3/EIRENE [27] has been used to obtain the plasma parameter range expected in the divertor region of $\mathrm{W} 7-\mathrm{X}$ for two different plasma regimes: the attached and partially detached plasma in a standard magnetic configuration. For the study we select the partially detached plasma regime with electron density reaching $10^{14} \mathrm{~cm}^{-3}$. Figure $2(\mathrm{~b}$ and $\mathrm{c})$ shows radial profiles of electron density and electron temperature used for the simulation of the beam penetration. In Figure 2 (a) the resulting profiles of level populations relative to the initial (at the nozzle position) ground level population are plotted. Dashed vertical lines indicate the radial extension of interest where the beam line radiation of the three detected spectral lines should be visible. The orange curve (labelled with " $23 \mathrm{~S}$ relax") is a measure of the beam relaxation: it provides the ratio of the relative (to the ground state) populations of the metastable level $2^{3} \mathrm{~S}$ calculated by the steady-state and the time-dependent solution. A value close to one over the entire radial range of interest indicates the applicability of the steady-state CR solution. The population of the radiating levels strongly increases at $\mathrm{T}_{\mathrm{e}} \approx 5 \mathrm{eV}$ so that the beam starts to become visible in this region (for measurements at lower temperatures one could think of admixing other species with lower excitation energies like neon). The penetration depth of the visible beam is limited by ionisation losses of the beam atoms and amounts to around $2 \mathrm{~cm}$. (In the next step we will account for the charge exchange collisions with protons as well as elastic collisions with protons and neutrals, which might influence the beam penetration and divergence [28].) The surprisingly deep penetration at these high electron densities results from the low temperature moderating the ionisation losses.

\section{Accuracy of the reconstructed plasma parameters}

In this section we assess the applicability of the helium beam diagnostic at the high density and low temperature plasma by use of the Bayesian approach. We consider a single point in the beam corresponding to one observation volume (cross section of the beam and an observation line of typical radial extension of few millimetres) with hypothetic plasma parameters of $n_{e}=10^{14} \mathrm{~cm}^{-3}$ and $T_{e}=5 \mathrm{eV}$ (referred to as the detached plasma parameters). We calculate the expected ratios of spectral lines emitted by the beam atoms in this volume. Due to the beam relaxation the line ratios can be calculated for this selected fraction of the beam regardless of the plasma conditions along the path of the consid- 
ered beam atoms through the plasma. The Bayesian inversion of the simulated line ratios provides the probability distributions for $\mathrm{n}_{\mathrm{e}}$ and $\mathrm{T}_{\mathrm{e}}$. The uncertainties of the rate coefficients are incorporated in form of Gaussian priors with the widths extracted from the ADAS [29] data set helike_hps02he.dat (based on [30]). Furthermore, in this analysis we assume to have no additional knowledge on $n_{e}$ and $\mathrm{T}_{\mathrm{e}}$, therefore we take uniform prior distributions for $\mathrm{n}_{\mathrm{e}}$ and $\mathrm{T}_{\mathrm{e}}$.

In a first step, we assume perfect knowledge about the rate coefficients and simulate the situation of measuring two standard line ratios $(668 / 728 \mathrm{~nm}$ and $728 / 706 \mathrm{~nm})$ with a relative measurement error of $5 \%$, which is the minimum relative uncertainty of line intensity measurement we believe to achieve for this diagnostic. For this case, we obtain relatively large $n_{e} / T_{e}$ errors (standard deviations of the 1dimensional marginal posterior distributions) of $43 \% / 14 \%$, respectively (see Table 1 ). This is caused by the lower sensitivity of the line ratios to the plasma parameters in this parameter regime. Ratios of other possible lines were checked as well, with smallest $n_{e} / T_{e}$ errors of $48 \% / 15 \%$ for the line ratios $501 / 728 \mathrm{~nm}$ and $728 / 706 \mathrm{~nm}$. When we additionally include uncertainties of all rate coefficients, the $n_{e} / T_{e}$ errors increase in the case of the standard line ratios to $128 \% / 45 \%$. The large errors show that the measurement method is less suitable for the detached plasma parameters.

Table 1. Uncertainties of derived values of $n_{e}$ and $T_{e}$ for the case of measuring two line ratios (relative measurement error: 5\%; RCs: rate coefficients).

\begin{tabular}{|c|c|c|}
\hline & $\sigma\left(\mathrm{n}_{\mathrm{e}}\right)$ & $\sigma\left(\mathrm{T}_{\mathrm{e}}\right)$ \\
\hline $\begin{array}{c}\text { RCs: perfect knowledge } \\
\text { Fit line ratios: }\end{array}$ & $43 \%$ & $14 \%$ \\
$667 / 728$ and 728/706 & & \\
\hline RCs: perfect knowledge & $48 \%$ & $15 \%$ \\
Fit line ratios: & & \\
501/728 and 728/706 & & \\
\hline RCs: uncertain & $128 \%$ & $45 \%$ \\
\hline
\end{tabular}




\begin{tabular}{|c|c|c|}
\hline Fit line ratios: & & \\
$667 / 728$ and $728 / 706$ & & \\
\hline
\end{tabular}

\section{Analysis of absolute line intensities}

The probabilistic model we use allows us in an easy way to study the impact of a changed experimental setup on the diagnostic accuracy. In the next step we investigate the additional use of the information which is contained in the absolute line intensities. This implies extending the diagnostic calibration to include not only a relative intensity calibration of the observation system at the three wavelengths of concern, but additionally its absolute intensity calibration.

Again, we first simulate the impact of the changed experimental setup on the diagnostic accuracy including only measurement errors (starting with the relative uncertainty of the absolute intensity of each line of 5\%). The rate coefficients are treated as known model parameters. The $n_{e} / T_{e}$ measurement accuracy achieved by using absolute emission intensities is drastically increased to $14 \% / 1.2 \%$ (see Table 2). This is due to the strong dependence of the absolute line intensities on the plasma parameters, in contrast to the line intensity ratios. However, in this simulation perfect knowledge about the number of ground level helium atoms was assumed, which cannot be gained in practice. There are three main factors influencing this value: the gas flow through the nozzle, the geometric beam divergence and the beam attenuation on its penetration path due to e.g. ionisation losses of the beam atoms. The first two factors do not depend on the plasma and hence can be measured by appropriate calibration in a test bed. The beam ionisation, depending on the plasma parameters themselves, is negligible in the example simulation of Figure 2 in the first half of the visible beam path $(\mathrm{r}<1.8 \mathrm{~cm})$. In the second half the beam attenuation can be estimated by the time-dependent CR model calculation using $n_{e} / T_{e}$ profile information obtained for the radial positions between the plasma edge and the evaluated observation point. In order to assess the impact of the fact that the beam attenuation is uncertain (e.g. due to unknown rate coefficients for beam atom losses caused by charge exchange collisions with pro- 
tons) we introduce it as an additional uncertain (nuisance) parameter into the probabilistic model by a Gaussian prior distribution of the number of beam atoms in the (hypothetic) observation volume. The maximum value of the prior results from the atom density distribution in the beam (to be measured on a test bed), the cross section with the observation line and the beam attenuation due to its interaction with the plasma. For our analysis, we can set the maximum value equal to one since the analysis does not depend on this value. For the relative standard deviation of the Gaussian prior we choose the value of 0.5 . This accounts for the assumed relative uncertainty of $50 \%$ of the number of beam atoms in the observation volume (mostly due to the uncertain beam attenuation). Including this prior in the analysis increases the $n_{e} / T_{e}$ errors to $18 \% / 7 \%$ affecting significantly only the $T_{e}$ error. Finally, we account in the simulation again for the uncertainties of the rate coefficients. This further increases $n_{e} / T_{e}$ errors to $66 \% / 8 \%$. The $\mathrm{T}_{\mathrm{e}}$ error changes only marginally and remains at a relatively low level of $8 \%$. This seems to be determined by the strong dependence of the line intensities on $T_{e}$ and on the excitation rate coefficients from the ground state which are known with higher precision than the rate coefficients for population transfer between the excited levels.

Table 2. Uncertainties of derived values of $n_{e}$ and $T_{e}$ for the case of measuring absolute intensities of three lines (667.8 nm, $706.5 \mathrm{~nm}$ and $728.1 \mathrm{~nm}$, relative measurement error of the line intensities: $5 \%$ ).

\begin{tabular}{|c|c|c|}
\hline & $\sigma\left(\mathrm{n}_{\mathrm{e}}\right)$ & $\sigma\left(\mathrm{T}_{\mathrm{e}}\right)$ \\
\hline $\begin{array}{c}\text { RCs: perfect knowledge } \\
\text { Beam attenuation: } \\
\text { perfect knowledge }\end{array}$ & $14 \%$ & $1.2 \%$ \\
\hline $\begin{array}{c}\text { RCs: perfect knowledge } \\
\text { Beam attenuation: }\end{array}$ & $18 \%$ & $7 \%$ \\
uncertain ( $\sigma=50 \%)$ & & \\
\hline RCs: uncertain & $66 \%$ & $8 \%$ \\
Beam attenuation: & & \\
\hline
\end{tabular}




\begin{tabular}{|l|l|l|}
\hline uncertain $(\sigma=50 \%)$ & & \\
\hline
\end{tabular}

As a last step we perform a sensitivity analysis concerning the measurement errors of the line intensities and an extension of the measured helium spectrum to more than three lines. In a case of larger measurement errors (relative error of $10 \%$ for each line intensity) the $n_{e}$ error strongly increases to $122 \%$ while $\mathrm{T}_{\mathrm{e}}$ error is hardly affected $(8.7 \%$, see Table 3$)$. This shows the high importance of a precise relative calibration of the observation system to obtain an even moderately accurate density measurement which can be slightly improved by extending the observation to more spectral lines, as the following study shows: Including the line at $501.6 \mathrm{~nm}$ while keeping the relative measurement errors of line intensities at the level of $10 \%$ reduces the $n_{e} / T_{e}$ errors to $107 \% / 8.5 \%$. Including two additional lines in the same spectral region $(492.2 \mathrm{~nm}$ and $504.8 \mathrm{~nm})$ further reduces the $\mathrm{n}_{\mathrm{e}} / \mathrm{T}_{\mathrm{e}}$ errors to $103 \% / 8 \%$.

The requirement for the absolute calibration is, however, much relaxed, as the number of beam atoms in the observation volume in the analysis is assumed to be known only to a precision of $50 \%$. This is equivalent to an uncertain overall absolute calibration factor. Therefore, enlarging the relative uncertainty of the absolute calibration factor to e.g. $20 \%$ while keeping the relative calibration between different wavelengths at $5 \%$ only marginally enhances the resulting $n_{e}$ and $T_{e}$ errors when comparing to the much more rigorous case of keeping also the absolute calibration factor uncertainty at $5 \%$ or $10 \%$. This is of practical interest, because the absolute calibration is expected suffer from unknown transmission losses due to window coating by plasma deposition.

Table 3. Uncertainties of derived values of $n_{e}$ and $T_{e}$ for the case of measuring more than three lines with lower relative accuracy of line intensities of $10 \%$ and uncertain beam attenuation $(\sigma=50 \%)$.

\begin{tabular}{|c|c|c|}
\hline & $\sigma\left(\mathrm{n}_{\mathrm{e}}\right)$ & $\sigma\left(\mathrm{T}_{\mathrm{e}}\right)$ \\
\hline RCs: uncertain & $66 \%$ & $8 \%$ \\
\hline
\end{tabular}




\begin{tabular}{|c|c|c|}
\hline $\begin{array}{c}\text { Fit abs. intensities of: } \\
667,706 \text { and } 728\end{array}$ & \\
Rel. measurement error: 5\% & & \\
\hline RCs: uncertain & $122 \%$ & $8.7 \%$ \\
Fit abs. intensities of: & & \\
667,706 and 728 & & \\
Rel. measurement error: $10 \%$ & & \\
\hline RCs: uncertain & & \\
Fit abs. intensities of: & & \\
$667,706,728$ and 501 & & \\
Rel. measurement error: $10 \%$ & & \\
\hline RCs: uncertain & & \\
Fit abs. intensities of: & & \\
$667,706,728,501,492$ and 504 & & \\
Rel. measurement error: $10 \%$ & & \\
\hline
\end{tabular}

As mentioned, the simulations described above are considering the inversion of local plasma parameters at a single spatial position along the beam. As next step, we are planning to extend the model to the entire helium beam propagating through the plasma. This will allow for the calculation of the adverse impact of the beam attenuation and divergence on the signal-to-noise ratio when considering absolute photon fluxes in the analysis. The resulting full radial profile of the uncertainties of $n_{e}$ and $T_{e}$ will allow us to estimate the required dynamic range of the detector.

\section{Refining the beam excitation model}

As measurements at TEXTOR have shown [1], the agreement that can be achieved between the plasma parameters obtained from helium beam emission spectroscopy and independent diagnostics is 
on the level of $10 \%$ for $n_{e}$ and $30 \%$ for $T_{e}$. This is a better agreement than expected when taking into account the uncertainty of the rate coefficients as found in the ADAS dataset. We performed a sensitivity analysis as described above for the $\mathrm{n}_{\mathrm{e}}$ and $\mathrm{T}_{\mathrm{e}}$ measurement from line intensity ratios at standard edge fusion plasma parameters $\left(\mathrm{T}_{\mathrm{e}}=32 \mathrm{eV}, \mathrm{n}_{\mathrm{e}}=4 \times 10^{12} \mathrm{~cm}^{-3}\right)$. The errors obtained this way are $50 \%$ for $\mathrm{n}_{\mathrm{e}}$ and $26 \%$ for $\mathrm{T}_{\mathrm{e}}$. They are dominantly caused by the uncertainty of the rate coefficients, rather than the experimental uncertainties of the intensity measurements. By the fact, that the agreement for the $\mathrm{n}_{\mathrm{e}}$ measurement achieved in practice is clearly better than our prediction, it can be seen, that the density reconstruction can be improved by taking into account additional information from other diagnostics. The probabilistic approach we are using allows performing this task by obtaining a set of refined rate coefficients, suitable to give a better description of the emission data than original set of rate coefficients.

In the following it is shown, how an overall agreement as observed at TEXTOR [1] allows for the statement of refined error margins of some of the rate coefficients given in ADAS. The complete set of measured data from the range of experiments from which our example result is concluded could be analysed in a similar way and would allow obtaining more detailed information. In order to show the potential of the method, we introduce the additional information to our probabilistic model by using Gaussian priors for $\mathrm{n}_{\mathrm{e}}$ and $\mathrm{T}_{\mathrm{e}}$ with the maxima at $\mathrm{n}_{\mathrm{e}}=4 \times 10^{12} \mathrm{~cm}^{-3}$ and $\mathrm{T}_{\mathrm{e}}=32 \mathrm{eV}$ (as measured by the TEXTOR helium beam) and relative standard deviations of $10 \%$ and $30 \%$, respectively (corresponding to the believed accuracy of the helium beam, see [1]). By this, it is possible to refine two rate coefficients $1{ }^{1} \mathrm{~S} \rightarrow 3^{1} \mathrm{~S}$ and $3^{1} \mathrm{~S} \rightarrow 3^{1} \mathrm{P}$ of transitions directly influencing the population of the upper level ${ }^{1} \mathrm{~S}$ of one of the three standard lines at $728 \mathrm{~nm}$. The relative uncertainty of these two coefficients without refinement (encoded in the priors) is $11 \%$ and $30 \%$, respectively, according to the ADAS data set helike_hps02he.dat. The 2D posterior for these two rate coefficients, is again calculated on a grid as described in Section 3 by marginalisation of the posterior over $n_{e}, T_{e}$ and all rate coefficients except for the two of interest. The marginal posterior is plotted in Figure 3. Its maximum is located at unchanged values of the rate coefficients. This is reasonable since we assumed agreement between the helium beam diagnostic and the validating diagnostics, i.e. the prior maxima of $\mathrm{n}_{\mathrm{e}}$ and $\mathrm{T}_{\mathrm{e}}$ were lying at 
the original values for $n_{e}$ and $T_{e}$ used for the simulation of the measured line ratios. In contrast, the standard deviations of resulting $1 \mathrm{D}$ posteriors for both rate coefficients $9.9 \%$ and $18.2 \%$ are lower than the ones of the corresponding input priors based on the ADAS data set. Especially the uncertainty of the second rate coefficient $\left(3{ }^{1} \mathrm{~S} \rightarrow 3{ }^{1} \mathrm{P}\right)$ is significantly lowered (30\% to $\left.18.2 \%\right)$. In addition, the combined uncertainty of both rate coefficients is even slightly smaller, because there is a correlation between both rate coefficients as is visible on the $2 \mathrm{D}$ posterior.

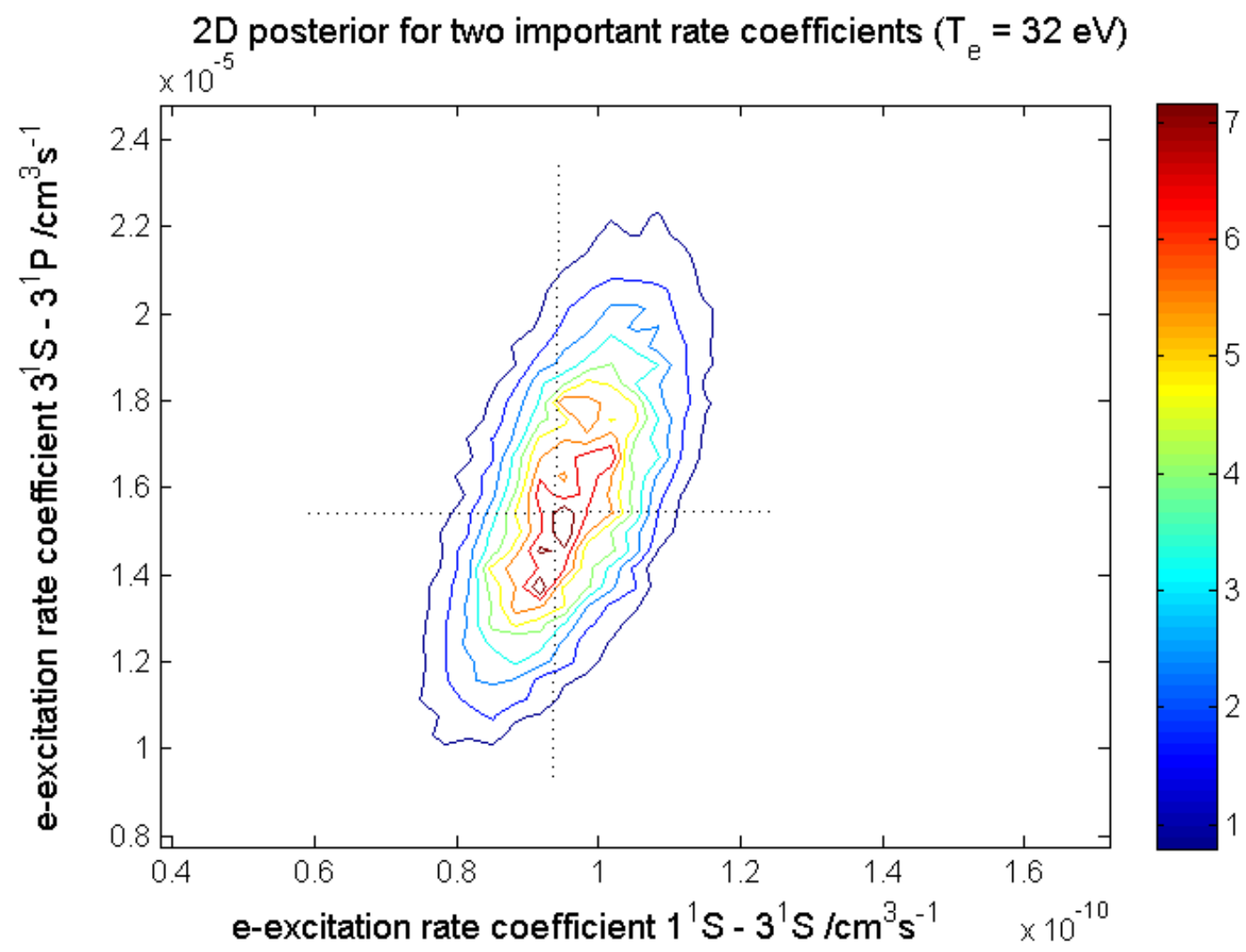

Figure 3: Contour plot of the posterior distribution of two rate coefficients important for the modelling of the line intensity at $728 \mathrm{~nm}$.

These results obtained by a probabilistic data analysis of comparative measurements of helium beam and other diagnostics should be considered as effective coefficients of total collisional population transfer between the respective levels rather than coefficients of electron collisional excitation. This is because we do not distinguish between electron and e.g. proton collisions which could play a significant role in the population transfer between levels of similar excitation energy [31]. Moreover, there might be other processes which are not included in our model, such as deviations of the Maxwellian 
energy distribution of electrons [3]. The obtained results should be considered as an improved atomic data set for modelling of helium beams applied to diagnose plasmas of similar parameters/constituents.

\section{Summary}

Thermal and supersonic helium beams are established diagnostics for measurement of electron density and temperature of the plasma edge of fusion experiments. The accuracy of the method is considerably influenced by the uncertainties of the rate coefficients for collisional excitation. The probabilistic approach we use allows us to asses quantitatively the final measurement errors including many disparate uncertainty sources. We apply this model for the study of the suitability of the measurement method for a different plasma parameter range: a high density $\left(\mathrm{n}_{\mathrm{e}}=10^{14} \mathrm{~cm}^{-3}\right)$ and low temperature $\left(\mathrm{T}_{\mathrm{e}}=5 \mathrm{eV}\right)$ divertor plasma as expected in the partial detached regime of the W7-X stellarator. The resulting $\mathrm{T}_{\mathrm{e}}$ error can be strongly reduced (to $\sim 8 \%$ ) by measuring and analysing absolute line intensities instead of their ratios even in the case of uncertain beam attenuation which must be estimated from the beam propagation through the plasma. An exact relative (for different wavelengths) calibration of the observation system to the level of $\sim 5 \%$ and absolute calibration to the level of $\sim 20 \%$ is crucial to keep the $n_{e}$ error below $70 \%$. The probabilistic approach can be applied to refine the atomic data set of helium beams by utilising comparisons of $n_{e}$ and $T_{e}$ measurement by helium beams and other diagnostics. 


\section{References}

[1] O Schmitz, IL Beigman, LA Vainshtein, B Schweer, M Kantor, A Pospieszczyk, Y Xu, M Krychowiak, M Lehnen, U Samm, B Unterberg and the TEXTOR team, Plasma Phys. Control. Fusion 50 (2008) 115004.

[2] S Kajita, N Ohno, S Takamura, T Nakan, PHYSICS OF PLASMAS 13 (2006) 013301.

[3] M Brix, PhD Thesis Ruhr-Universitat Bochum (1999 Forschungszentrum Jülich Report Jül-3638).

[4] CF Burrell and H-J Kunze, Phys. Rev. A 18 (1978) 2081-2088.

[5] R Denkelmann, S Maurmann, T Lokajczyk, P Drepper, H-J Kunze, J. Phys. B: At. Mol. Opt. Phys. 32 (1999) 4635-46.

[6] M Krychowiak, Ph Mertens, R Konig ,B Schweer, S Brezinsek, O Schmitz, M Brix, U Samm, T Klinger, Plasma Phys. Control. Fusion 50 (2008) 065015.

[7] M Krychowiak, Ph Mertens, R König, B Schweer, S Brezinsek, O Schmitz, M Brix, U Samm, R Wolf, T Klinger, J. Phys.: Conf. Ser. 227 (2010) 012024.

[8] Y Andrew and MG O’Mullane, Plasma Phys. Control. Fusion 42 (2000) 301-307.

[9] N Ramasubramanian, R König, Y Feng, L Giannone, P Grigull, T Klinger, K McCormick, H Thomsen, U Wenzel and the W7-AS Team, Nucl. Fusion 44 (2004) 992-998.

[10] M Otte, J Belapure, E Chlechowitz, M Glaubitz, G Hasinger, B Huber, M Krychowiak, HP Laqua, S Marsen, E Müller, DM Schlosser, T Stange, F Wagner, A Weller, R Wolf, and D Zhang, 37th EPS Conf. on Contr. Fusion and Plasma Physics (2010).

[11] M Brix and B Schweer, 24th EPS Conf. on Contr. Fusion and Plasma Physics (1997) 1837-1840.

[12] GA Cottrell, Maximum entropy and plasma physics, in: Maximum Entropy in Action: A Collection of Expository Essays, edited by B Buck and VA Macaulay, Oxford: Clarendon Press, (1991).

[13] R Fischer and V Dose, Plasma Phys. Contr. Fusion 41 (1999) 1109. 
[14] AP Millar, DC McDonald, and DA Diver, Plasma Phys. Control. Fusion 42 (2000) 337.

[15] R Fischer, A Dinklage, and E Pasch, Plasma Phys. Control. Fusion 45 (2003) 1095.

[16] A Dinklage, R Fischer, and J Svensson, Fusion Science and Technology 46 (2004) 355.

[17] J Svensson, A Dinklage, J Geiger, A Werner, and R Fischer, Rev. Sci. Instrum. 75 (2004) 4219.

[18] J Svensson and A Werner, Plasma Phys. Control. Fusion 50 (2008) 085002.

[19] D Dodt, A Dinklage, R Fischer, K Bartschat, O Zatsarinny, D Loffhagen, J. Phys. D: Appl. Phys. $41(2008) 205207$.

[20] M Reginatto, and A Zimbal, Rev. Sci. Instrum. 79 (2008) 023505.

[21] OP Ford, J Svensson, M Beurskens, A Boboc, J Flanagan, M Kempenaars, DC McDonald and JET EFDA contributors, 36th EPS Conf. on Contr. Fusion and Plasma Physics (2009).

[22] D Dodt, A Dinklage, K Bartschat, O Zatsarinny, New J. Phys. 12 (2010) 073018.

[23] MJ Hole, G von Nessi, J Bertram, J Svensson, LC Appel, BD Blackwell, RL Dewar and J Howard, J. Plasma Fusion Res. SERIES 9 (2010) 479.

[24] D Sivia and J Skilling, Data Analysis: A Bayesian Tutorial, Second Edition, Oxford Uni-versity Press, Oxford, 2006.

[25] A Gelman, JB Carlin, HS Stern, DB Rubin, Bayesian data analysis, Texts in statistical science, Chapman \& Hall/CRC, Boca Raton, Fla., 1995.

[26] R König, J Baldzuhn, W Biel, C Biedermann, R Burhenn, S Bozhenkov, J Cantarini, H Dreier, M Endler, H-J Hartfuss, D Hildebrandt, M Hirsch, M Jakubowski, R Jimenez-Gomez, G Kocsis, P Kornejew, M Krychowiak, H P Laqua, M Laux, JW Oosterbeek, E Pasch, T Richert, W Schneider, B Schweer, J Svensson, H Thomsen, A Weller, A Werner, R Wolf, D Zhang, S Zoletnik, Diagnostics Design for Steady-State Operation of the W7-X Stellarator, accepted for publication by Rev. Sci. Instrum. 
[27] Y Feng, F Sardei, J Kisslinger, J. Nucl. Mat. 266-269 (1999) 812.

[28] A Hidalgo, FL Tabarés, D Tafalla, B Brañas, 32th EPS Conf. on Contr. Fusion and Plasma Physics (2005) P-2.084

[29] HP Summers and MG O'Mullane, The Atomic Data and Analysis Structure, In R.E.H. Clark and D.H. Reiter (eds.), editors, Nuclear Fusion Research. Understanding Plasma-Surface Interactions. Springer-Verlag, Berlin, 2005.

[30] YuV Ralchenko, RK Janev, T Kato, DV Fursa, I Bray, and FJ de Heer. Cross Sections Database for Collision Processes of Helium Atom with Charged Particles. I. Electron Impact Processes. Rep. NIFS-DATA-59, NIFS, Nagoya, 2000.

[31] M Krychowiak, Laser-induced fluorescence of atomic helium beams in a fusion edge plasma, IPP report 13/6 of the Max-Planck-Institute for Plasma Physics, 2007. 